\title{
Chasing the Ghost of Imaging Remission in Rheumatoid Arthritis
}
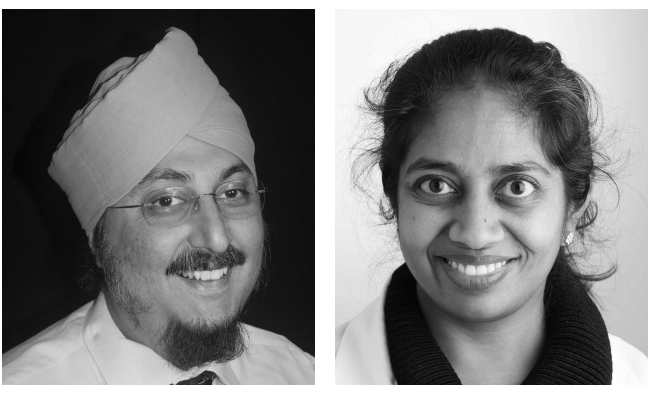

The role of musculoskeletal ultrasound (MSK-US) in rheumatoid arthritis (RA) is evolving, albeit with twists and turns. Early RA randomized clinical studies have demonstrated little added value of MSK-US when used prospectively in a treat-to-target strategy ${ }^{1,2}$. Recent editorials have discussed the issues of clinical trial design and standardization US measures raised by these studies ${ }^{3,4}$.

Ostensibly the goal of therapy in RA is to achieve remission and hence halt progression of erosions, as well as to maintain function. However, sustained remission is not obtained in the majority of patients with established $\mathrm{RA}^{5}$. Further, despite more stringent composite and Boolean remission criteria, progression of erosions still occurs regardless of applied clinical remission criteria ${ }^{6,7,8}$.

Progression of erosions in subjects in remission is considered a result of subclinical synovitis. MSK-US can be used at the bedside to gauge residual synovitis in many joint areas. In metaanalyses of heterogeneous RA populations in clinical remission, the prevalence of greyscale and Doppler-positive subjects was estimated at $43 \%{ }^{9}$. Residual synovitis prevalence was comparable even when using alternate definitions of remission. Power Doppler seems to be the key component of imaging synovitis linked to progression of erosions ${ }^{9,10}$. Of note in the 4 studies included for structural progression, definition of progression ranged from change of total Sharp score of greater than zero to greater than the smallest detectable difference ${ }^{9,10}$. In addition, prevalence of residual synovitis was higher in patients with longstanding RA compared to early RA. One drawback of these metaanalyses is that they did not analyze subjects who were taking solely biologic agents compared to standard disease-modifying antirheumatic drugs (DMARD). Limited studies using observational cohorts have suggested that the rate of progression of erosions may be slower in patients using biological DMARD compared to conventional ones ${ }^{11}$.

Metaanalyses have also suggested that residual power Doppler is associated with higher risk of flare ${ }^{9,10}$. Although small observational studies have suggested that longer duration of remission is linked to decreased risk of flare, the metaanalysis by Han, et al could not confirm this because of high heterogeneity of the included studies ${ }^{10,12}$. The use of MSK-US in the prediction of sustained remission is of imminent importance owing to significant interest in conducting biologic de-escalation clinical trials to reduce the medical economic burden during these fiscally challenging times. Lamers-Karnebeek, et al reported on MSK-US in a 1-year prospective study of patients with RA in low disease activity or remission who stopped antitumor necrosis factor agents. MSK-US could not predict flare on an individual level ${ }^{13}$. Thus, the 2 main reasons for MSK-US in patients with RA in remission are for prevention of damage from ongoing synovitis and prediction of flare, especially when medication de-escalation needs to be considered.

In this issue of The Journal, Zufferey, et al report a multicenter retrospective study of an interesting concept: evaluating MSK-US residual synovitis in predicting loss of remission in patients in clinical remission based on the 28-joint Disease Activity Score using erythrocyte sedimentation rate (DAS28-ESR) $<2.6^{14}$. The authors conclude that loss of remission was noted in $60 \%$ of 378 remission phases, where $66 \%$ of these had DAS28-ESR $>2.6$ and $23 \%$ started new DMARD more than 60 days from the last MSK-US examination. In addition, they report that the presence of residual US synovitis was associated with shorter durations of clinical remission and that residual US synovitis seen early in the remission period (3-6 mos) predicted higher risk for loss of remission. While the results of this study were of significant interest, it is important to consider some of the caveats. DAS28-ESR is considered one of the least stringent clinical remission measures ${ }^{15}$. Use of more stringent composite or Boolean measures may have resulted in lower rates of loss of clinical remission and changed the conclusions. The MSK-US scoring system used is somewhat atypical because it only scores B-mode at the volar aspects of the metacarpophalangeal (MCP) and proximal interphalangeal (PIP) joints, while power Doppler is scored at the

\section{See US predicting remission in RA, page 887}

Personal non-commercial use only. The Journal of Rheumatology Copyright (c) 2018. All rights reserved. 
dorsal aspects of these joints ${ }^{16}$. Thus, it is unclear whether this scoring system is translatable to the common busy rheumatologist's clinic. There were several definitions for residual synovitis by MSK-US as well as definitions of loss of clinical remission, and this provides some ambiguity to the outcome and difficulty in translating to the clinical setting. The duration of clinical remission was imputed in 2 different ways (left mode and right mode), while the truth lies somewhere in between. Last and most important, to demonstrate the added value of MSK-US in predicting loss of clinical remission, accounting for baseline DAS28-ESR is of significance in a retrospective clinical trial. Adjusting for baseline DAS28-ESR potentially could have altered the results.

US data were available for B-mode: 318 patients and 378 remission phases; Doppler: 292 patients and 292 remission phases; and in combined mode: 276 patients and 321 phases. Baseline characteristics in the residual synovitis positive and negative groups were similar except that duration of remission was higher in the residual synovitis-negative group. Of note, about two-thirds of all the patients were taking biologic agents at baseline and one-third were taking synthetic DMARD. Remarkably, 6\% (19) of patients were not taking DMARD. Residual synovitis by B-mode definition was seen in $32 \%$ of the cohort and in $58 \%$ using the combined definition. Patients with residual synovitis tended to be older and seropositive.

The analysis for duration of remission was heavily influenced by the MSK-US synovitis definition used. A difference of 2 to 5 months' duration of clinical remission between residual MSK-US positive and negative groups was seen only when applying the combined US definition of synovitis. In addition, left mode imputation (i.e., patients not in remission) may also have inflated the differences between the groups. Similarly, adjusted HR were only significant when using the combined synovitis score. An HR of $1.5(1.1-2.1)$ was calculated for patients with combined MSK-US residual synovitis at baseline compared to those without using right mode imputation. Using right mode imputation for the early US subgroup, duration of remission was longer in the residual synovitis negative group when using the combined or Doppler synovitis definitions.

The strength of the aforementioned study is that it analyzes data collected in a registry, a practice that is likely to reflect a more diverse population. The overall conclusions from the report are that in patients in clinical remission, residual synovitis may be associated with decreased duration of remission, but the differences between the groups are not striking. Notable factors that may have affected the strength of the report include lack of standardization of equipment, Doppler sensitivity, and an atypical scanning method that may have inherently contributed to loss of dorsal MCP and PIP Doppler information. The majority of the sonographers were treating rheumatologists and were not blinded to the data, but that is in line with a real-world scenario. Further, alteration of therapy within 6 months of the MSK-US examination was allowed and not counted as loss of remission. This may have biased some of the residual synovitis group to stay in remission.

The current study suggests a role of MSK-US in predicting remission duration and implied applicability to considering biologic de-escalation. Central to the proposition of using MSK-US in patients with RA in remission is to prevent progression of erosions. However, this assumption needs to be confirmed in patients using biologic therapies. Further, the bar for erosion progression needs to be clinically relevant and functional, and patient-related outcomes also need to be considered.

GURJIT S. KAELEY, MRCP, RhMSUS, University of Florida College of Medicine, Jacksonville, Florida;

VEENA K. RANGANATH, MD, MS, RhMSUS, University of California at Los Angeles, Los Angeles, California, USA.

Address correspondence to Dr. G.S. Kaeley, University of Florida College of Medicine, Division of Rheumatology, PO Box 162, Ponte Vedra, Florida 32004, USA.E-mail: drgurj@gmail.com

\section{REFERENCES}

1. Haavardsholm EA, Aga AB, Olsen IC, Lillegraven S, Hammer HB, Uhlig T, et al. Ultrasound in management of rheumatoid arthritis: ARCTIC randomised controlled strategy trial. BMJ 2016;354:i4205.

2. Dale J, Stirling A, Zhang R, Purves D, Foley J, Sambrook M, et al. Targeting ultrasound remission in early rheumatoid arthritis: the results of the TaSER study, a randomised clinical trial. Ann Rheum Dis 2016;75:1043-50.

3. D'Agostino MA, Boers M, Wakefield RJ, Emery P, Conaghan PG. Is it time to revisit the role of ultrasound in rheumatoid arthritis management? Ann Rheum Dis 2017;76:7-8

4. Caporali R, Smolen JS. Back to the future: forget ultrasound and focus on clinical assessment in rheumatoid arthritis management. Ann Rheum Dis 2018;77:18-20.

5. Prince FH, Bykerk VP, Shadick NA, Lu B, Cui J, Frits M, et al. Sustained rheumatoid arthritis remission is uncommon in clinical practice. Arthritis Res Ther 2012;14:R68.

6. Klarenbeek NB, Koevoets R, van der Heijde DM, Gerards AH, Ten Wolde S, Kerstens PJ, et al. Association with joint damage and physical functioning of nine composite indices and the 2011 ACR/EULAR remission criteria in rheumatoid arthritis. Ann Rheum Dis 2011;70:1815-21.

7. Lillegraven S, Prince FH, Shadick NA, Bykerk VP, Lu B, Frits ML, et al. Remission and radiographic outcome in rheumatoid arthritis: application of the 2011 ACR/EULAR remission criteria in an observational cohort. Ann Rheum Dis 2012;71:681-6.

8. Sewerin P, Vordenbaeumen S, Hoyer A, Brinks R, Buchbender C, Miese F, et al. Silent progression in patients with rheumatoid arthritis: is DAS28 remission an insufficient goal in RA? Results from the German Remission-plus cohort. BMC Musculoskelet Disord 2017;18:163

9. Nguyen H, Ruyssen-Witrand A, Gandjbakhch F, Constantin A, Foltz V, Cantagrel A. Prevalence of ultrasound-detected residual synovitis and risk of relapse and structural progression in rheumatoid arthritis patients in clinical remission: a systematic review and meta-analysis. Rheumatology 2014;53:2110-8.

Personal non-commercial use only. The Journal of Rheumatology Copyright @ 2018. All rights reserved. 
10. Han J, Geng Y, Deng X, Zhang Z. Subclinical synovitis assessed by ultrasound predicts flare and progressive bone erosion in rheumatoid arthritis patients with clinical remission: a systematic review and metaanalysis. J Rheumatol 2016;43:2010-8.

11. Ciubotariu E, Gabay C, Finckh A, Physicians of the Swiss Clinical Quality Management Program for Rheumatoid Arthritis. Joint damage progression in patients with rheumatoid arthritis in clinical remission: do biologics perform better than synthetic antirheumatic drugs? J Rheumatol 2014;41:1576-82.

12. Tokai N, Ogasawara M, Gorai M, Matsuki Y, Yamada Y, Murayama $\mathrm{G}$, et al. Predictive value of bone destruction and duration of clinical remission for subclinical synovitis in rheumatoid arthritis patients. Mod Rheumatol 2015;25:540-5.

13. Lamers-Karnebeek FB, Luime JJ, Ten Cate DF, Teerenstra S, Swen $\mathrm{N}$, Gerards $\mathrm{AH}$, et al. Limited value for ultrasonography in predicting flare in rheumatoid arthritis patients with low disease activity stopping TNF inhibitors. Rheumatology 2017;56:1560-5.
14. Zufferey P, Scherer A, Nissen M, Ciurea A, Tamborrini G, Brulhart $\mathrm{L}$, et al, on behalf of the SONAR Group and the SCQM Foundation. Can ultrasound be used to predict loss of remission in RA patients in a real-life setting? A multicentre cohort study. J Rheumatol 2018;45:887-94.

15. Khanna D, Oh M, Furst DE, Ranganath V, Gold RH, Sharp JT, et al. Evaluation of the preliminary definitions of minimal disease activity and remission in an early seropositive rheumatoid arthritis cohort. Arthritis Rheum 2007;57:440-7.

16. Zufferey P, Brulhart L, Tamborrini G, Finckh A, Scherer A, Moller $\mathrm{B}$, et al. Ultrasound evaluation of synovitis in RA: correlation with clinical disease activity and sensitivity to change in an observational cohort study. Joint Bone Spine 2014;81:222-7.

J Rheumatol 2018;45:881-3; doi:10.3899/jrheum.180035 